Suzana de Magalhães Barreto

\title{
A Relação entre o Sistema de Gestão Baseado em Competências e o Contrato Psicológico do Indivíduo: Estudo de Caso em uma Empresa de Engenharia
}

Dissertação apresentada como requisito parcial para obtenção do título de Mestre pelo Programa de PósGraduação em Administração da PUC-Rio.

Orientador: José Roberto Gomes da Silva

Rio de Janeiro Novembro de 2007 
Suzana de Magalhães Barreto

\section{A Relação entre o Sistema de Gestão Baseado em Competências e o Contrato Psicológico do Indivíduo: Estudo de Caso em uma Empresa de Engenharia}

Dissertação apresentada como requisito parcial para obtenção do grau de Mestre pelo Programa de Pósgraduação em Administração de Empresas da PUCRio. Aprovada pela Comissão Examinadora abaixo assinada.

Prof. José Roberto Gomes da Silva

Orientador

Departamento de Administração - PUC-Rio

Profa. Sandra Regina da Rocha Pinto

Departamento de Administração - PUC-Rio

Profa. Maria Teresa Correia Coutinho

UFRJ - FACC

Prof. João Pontes Nogueira Vice-Decano de Pós-Graduação do CCS

Rio de Janeiro, 13 de novembro de 2007 
Todos os direitos reservados. É proibida reprodução total ou parcial do trabalho sem autorização da universidade, do autor e do orientador.

\section{Suzana de Magalhães Barreto}

Graduou-se em Engenharia de Produção Mecânica pela PUCRio em 2001. Ao se formar, foi contratada como analista de mercado na Telemar e, neste período, acumulou experiências na área de marketing, principalmente na área de Pesquisa de Mercado e Relacionamento com o Cliente. Em de 2003, trocou de empresa passando desde então a atuar na área de Gerenciamento de Projetos na Technip, multinacional francesa no ramo petrolífero.

Ficha Catalográfica

Barreto, Suzana de Magalhães

A relação entre o sistema de gestão baseado em competências e o contrato psicológico do indivíduo: estudo de caso em uma empresa de engenharia / Suzana de Magalhães Barreto ; orientador: José Roberto Gomes da Silva. - 2007.

118 f. : il. ; $30 \mathrm{~cm}$

Dissertação (Mestrado em Administração)-Pontifícia Universidade Católica do Rio de Janeiro, Rio de Janeiro, 2007.

Inclui bibliografia

1. Administração - Teses. 2. Gestão por competências. 3. Contrato psicológico. 4. Mudança organizacional. 5. Relações de trabalho. I. Silva, José Roberto Gomes da. II. Pontifícia Universidade Católica do Rio de Janeiro. Departamento de Administração. III. Título. 


\section{Agradecimentos}

Agradeço a todos que contribuíram na execução deste trabalho, direta e indiretamente, que mais que uma pesquisa, representou para mim o fim e o início de uma nova fase.

Em especial, agradeço ao meu orientador, que soube de uma maneira apropriada conduzir nosso trabalho, ao longo de um período de dúvidas e descobertas, transformando uma pergunta em uma obra.

Aos meus pais que sempre prezaram pela minha formação educacional, minha mãe com sua luz celestial e meu pai com seu suporte mais que especial. Às minhas irmãs, orgulhosas e corujas. Enfim, a toda minha família e amigos, presentes de onde quer que estejam me apoiando e fortificando sempre que foi preciso.

Ao meu companheiro, Ricardo, que por vezes me ajudou a restabelecer o caminho e seguir com persistência até o final.

Por final, aos entrevistados que acreditaram na contribuição a esta obra e viabilizaram interessantes descobertas. 


\section{Resumo}

Barreto, Suzana de Magalhães; da Silva, José Roberto Gomes (orientador). "A Relação entre o Sistema de Gestão Baseado em Competências e o Contrato Psicológico do Indivíduo: Estudo de Caso em uma Empresa de Engenharia". Rio de janeiro, 2008. 118p. Dissertação de Mestrado (Opção profissional) Departamento de Administração, Pontifícia Universidade Católica do Rio de Janeiro.

O presente trabalho buscou trazer compreensão sobre os principais aspectos que afetam o contrato psicológico que os indivíduos estabelecem com uma empresa que se encontra em processo de mudança nas relações de trabalho. Focalizou-se em um fenômeno característico da década atual, quando se verifica um aumento de empresas que buscam através da implantação de um sistema de gestão baseado em competências, condições para a obtenção de vantagem competitiva. No que diz respeito à gestão estratégica de recursos humanos, o conceito de competências é instrumento de referência para mediar as diferentes responsabilidades e contribuições individuais na empresa. Em contrapartida, o desempenho organizacional, dependerá da qualidade no grau de entrega dos indivíduos ao novo modelo de gestão, através da manutenção de um contrato psicológico positivo dos profissionais. Optou-se por desenvolver um estudo de caso qualitativo com características exploratórias. A organização escolhida foi a Technip S.A., empresa de engenharia que possui forte atuação no setor petroquímico e que se encontra em fase de implantação de um sistema de gestão baseado em competências. A metodologia utilizada na pesquisa de campo foi fundamentada em entrevistas individuais realizadas, por meio de questões semi-estruturadas. Buscou-se identificar a realidade social da organização a partir do estudo das expectativas dos indivíduos em relação à mesma, antes e depois da iniciativa de mudança, como forma importante de compreensão da estruturação da ação nesse ambiente. Os resultados conduziram à percepção da existência de uma relação de reciprocidade entre individuo e organização. Conclui-se que, enquanto a mudança exerce, sobre o contrato psicológico dos indivíduos, uma influência que vai desde o reforço ao rompimento, a leitura que os indivíduos fazem da mesma encontrase relacionada ao contrato psicológico pré-existente.

\section{Palavras Chaves}

Gestão por Competências; Contrato Psicológico; Mudança Organizacional; Relações de Trabalho 


\section{Abstract}

Barreto, Suzana de Magalhães; da Silva, José Roberto Gomes (advisor). "The Relationship between a Competence Management System and the Individual Psychological Contract: A case study of an engineering petrochemical enterprise". Rio de Janeiro, 2008. 118p. MSc. Dissertation (Professional Option) - Business Department, Pontifícia Universidade Católica of Rio de Janeiro.

The purpose of this study is to have a better understanding of the main aspects that affect the psychological contract established by individuals facing a changing process on their organizational work relationship. This study focused on the competence management implementation as a competitive advantage. In terms of resource management strategy, the concept of competence is an important instrument to measure different individual responsibilities and contributions to the organization. On the other hand, the organization performance will depend on how individuals interact with the management system in order to maintain a positive psychological contract. Therefore, a qualitative case study with exploratory characteristics was developed based on several interviews done with employees from a company that belongs to the petrochemical sector, called Technip S.A. This firm is implementing a competence management methodology. These interviews were based on previous questionnaires and their purpose was to identify the social reality of the organization based $o$ its individuals' expectation, before and after changing events. The main results of this study confirm a reciprocity relationship between individuals and organization, that a change in the work relationship can either reinforce the psychological contract or violate it, and the individuals' comprehension will directly depend on their pre-existent psychological contract. Finally, this study suggests that the success of a competence management system implementation depends on understanding the psychological contract of those involved on the changing events.

\section{Keywords}

Competences Management; Psychological Contract; Organizational Change; Work Relation 


\section{Sumário}

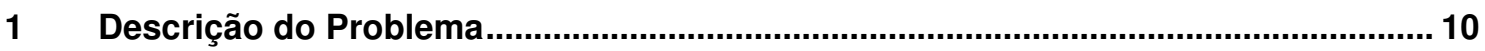

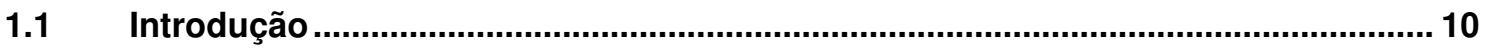

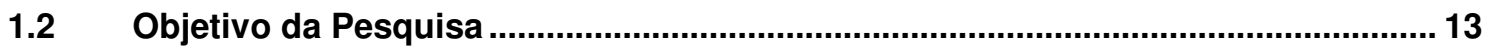

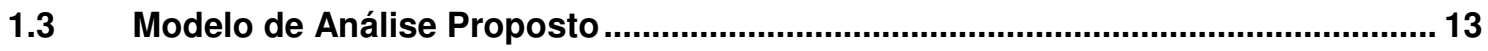

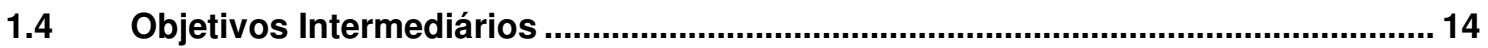

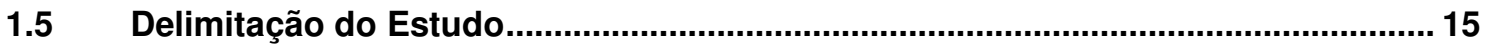

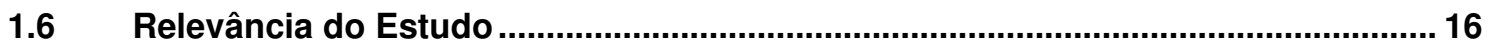

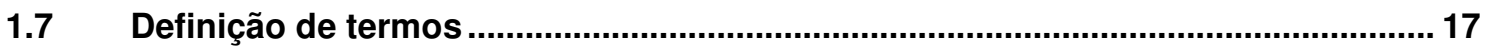

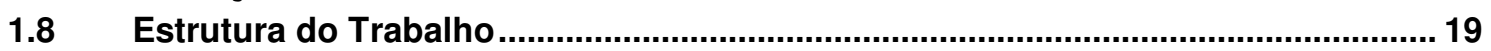

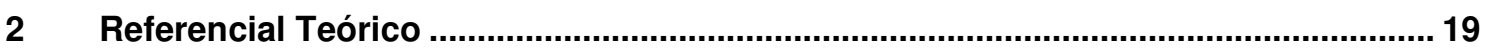

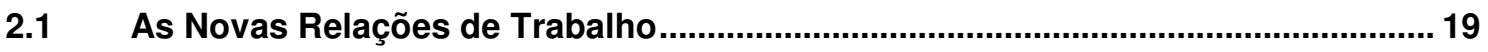

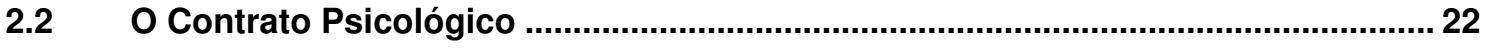

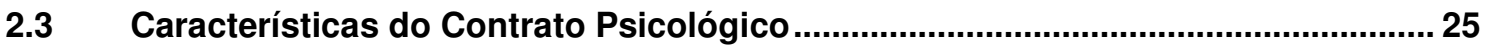

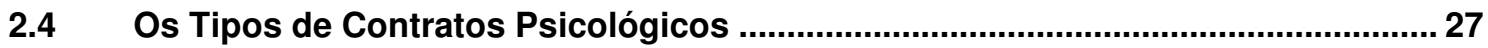

2.5 O Processo de Mudança do Contrato Psicológico ..................................................... 33

2.6 O processo de Ruptura e Violação do Contrato Psicológico..................................... 35

2.7 Mudanças no Estilo de Gestão de Pessoas ........................................................... 38

2.8 Gestão por Competências como um Estilo de Gestão ........................................... 39

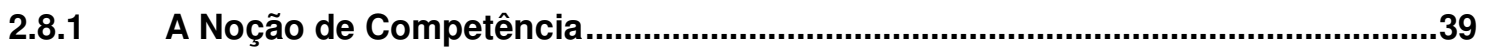

2.8.2 As dimensões da noção de competência no âmbito das organizações..............41

2.8.3 Entrega e Complexidade ......................................................................44

2.8.4 O Desenvolvimento e a Carreira Profissional na Gestão por Competências.....45

2.8.5 A Construção de um Sistema de Gestão do Desenvolvimento ............................48

2.8.6 A noção de competências no contexto organizacional.......................................48

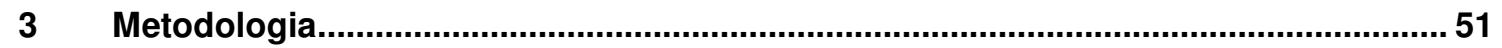

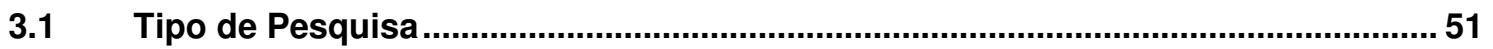

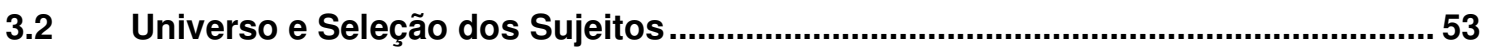

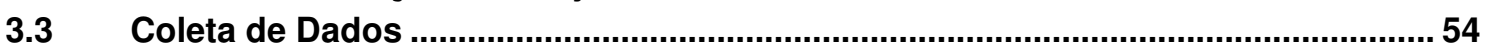

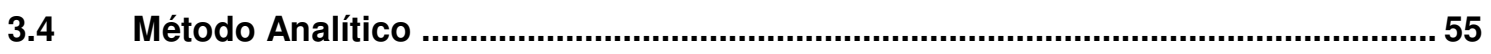

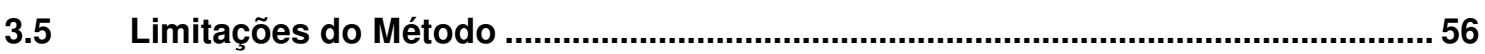

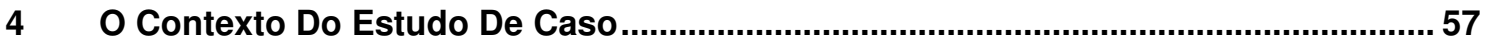

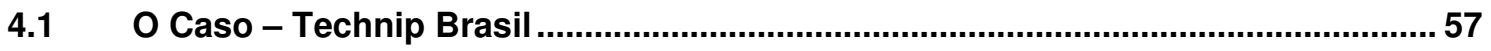

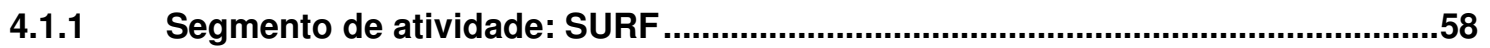

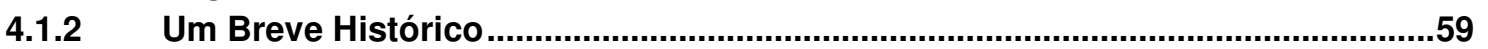

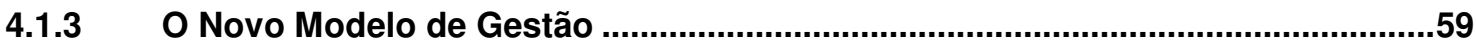

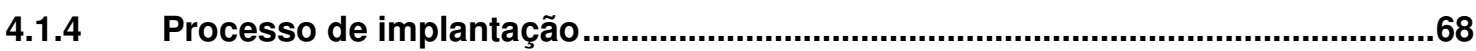

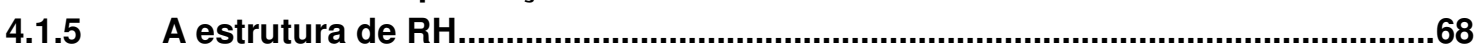

$5 \quad$ Resultados Das Entrevistas Com Profissionais ..................................................... 71

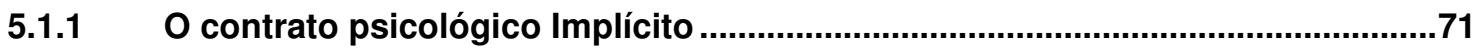

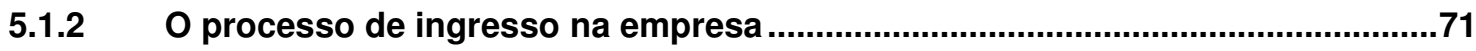

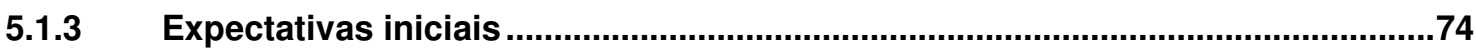

5.2 Grau de informação sobre o sistema e Percepção da mudança ............................. 77

5.2.1 Grau de informação sobre o conceito ...............................................................77 


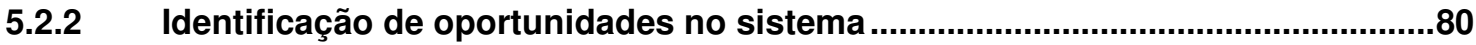

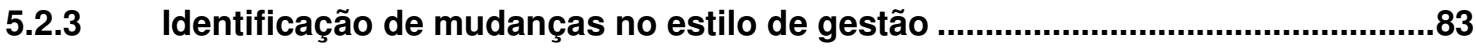

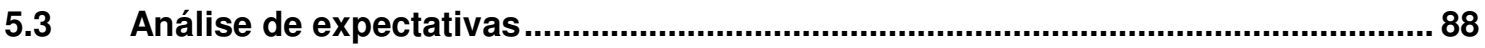

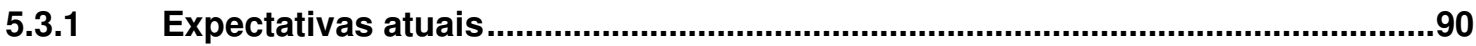

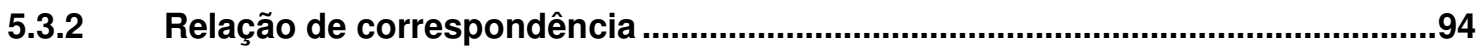

5.4 O processo de violação do contrato psicológico ..................................................... 97

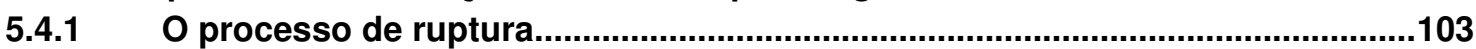

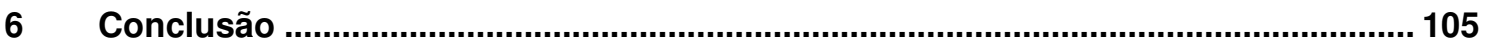

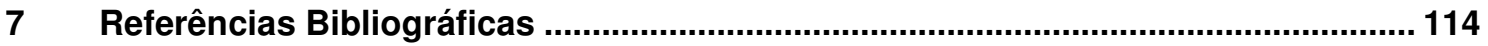




\section{Lista de Figuras}

Figura 1 - Modelo Relacional: Indivíduo x Organização x Gestão de Competências ........ 14

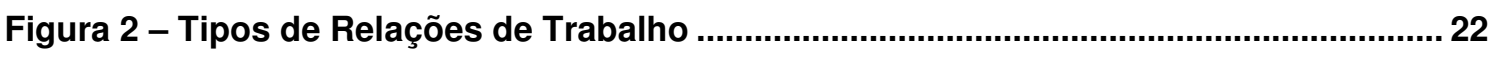

Figura 3 - Modelo Three-Tier Pyramid (pirâmide de três camadas) .................................... 30

Figura 4 - Fatores que afetam os Resultados ou a "Entrega" dos Indivíduos ..................... 38

Figura 5 - Exemplo de competência em ação na educação: elementos principais ............ 40

Figura 6 - Relação de Reciprocidade Mudança x Contrato Psicológico ............................ 111

\section{Lista de Quadros}

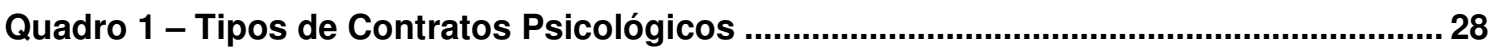

Quadro 2 - Características dos Contratos Psicológicos: 0 "velho" vs o "novo" ............... 31

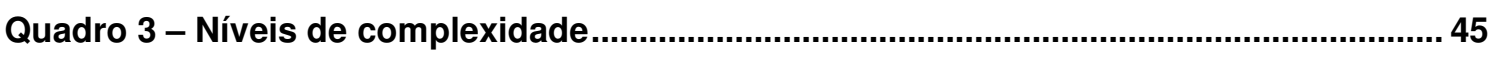

Quadro 4 - Situações Relevantes para Diferentes Estratégias de Pesquisa. .................... 52

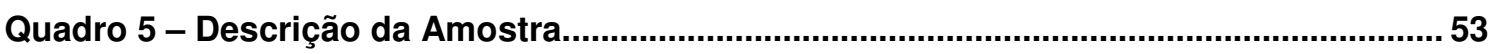

Quadro 6 - Evolução da implantação do novo modelo de gestão na Technip .....................61

Quadro 7 - Resumo dos fatores motivadores de entrada na empresa ................................ 73

Quadro 8 - Resumo dos aspectos citados......................................................................... 77

Quadro 9 - Resumo das associações sobre o sistema de gestão ........................................ 80

Quadro 10 - Resumo das percepções das oportunidades de melhoria ............................. 83

Quadro 11 - Resumo das mudanças identificadas no estilo de gestão da empresa .......... 87

Quadro 12 - Resumo dos motivos provocadores das mudanças ......................................... 87

Quadro 13 - Resumo das mudanças das expectativas atuais........................................... 94

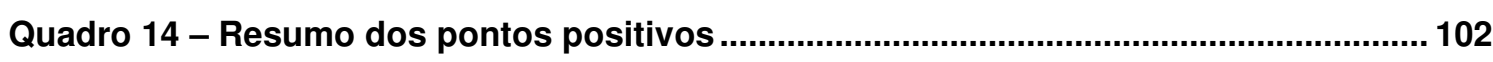

Quadro 15 - Resumo dos pontos negativos ................................................................... 103

Quadro 16 - Resumo dos motivos que levariam a deixar a relação com a empresa ....... 104

Quadro 17 - Roteiro de Análise de Influências............................................................... 113 